\title{
Multi-Jet Cross Sections in Charged Current Deep Inelastic Scattering at HERA
}

\author{
H. Wolfe * \\ University of Wisconsin, Madison - Department of Physics \\ Madison, Wisconsin - U.S.A.
}

\begin{abstract}
Differential jet cross sections have been measured in charged current deep inelastic $e p$ scattering at high boson virtualities $Q^{2}$ with the ZEUS detector at HERA using an integrated luminosity of $200 \mathrm{pb}^{-1}$. Jets were identified using the $k_{\top}$ cluster algorithm. Polarised and unpolarised-corrected cross sections are presented for inclusive-jet production as functions of $Q^{2}$, Bjorken $x$, jet transverse energy and jet pseudorapidity. The dijet invariant mass cross section is also presented. Observation of three-jet events in charged-current processes is reported for the first time. The predictions of a leadinglogarithm parton-shower Monte Carlo model are compared to the measurements.
\end{abstract}

\section{Introduction}

Jet production in charged-current (CC) deep inelastic ep scattering (DIS) provides a testing ground for QCD and for the electroweak sector of the Standard Model (SM). Up to leading order (LO) in the strong coupling constant, $\alpha_{S}$, jet production in CC DIS proceeds via the quark-parton model $(W q \rightarrow q)$, the QCD-Compton $(W q \rightarrow q g)$ and $W$-gluon fusion $(W g \rightarrow q \bar{q})$ processes. Thus, differential cross sections for jet production are sensitive to $\alpha_{S}$ and the mass of the propagator, $M_{W}$, which are fundamental parameters of the theory.

Since 2002, HERA has run with longitudinally-polarised electron or positron beams. In this paper, measurements are presented of inclusive-jet and dijet cross sections in $\mathrm{CC} e^{-} p$ DIS in the laboratory frame. Measurements of three-jet differential cross sections in CC DIS have been measured for the first time in $e p$ collisions. A small sample of four-jet events has also been observed in the data. The measurements are presented as functions of $Q^{2}, x$, the jet transverse energy, $E_{\mathrm{T}}^{\mathrm{jet}}$, and jet pseudorapidity, $\eta^{\mathrm{jet}}$. Predictions from a leading-logarithm parton-shower Monte Carlo (MC) model are compared to the measurements. Results for negative and positive longitudinally-polarised electron beams are also presented.

\section{Data selection and jet search}

The data were collected during the running period 2004-2006. Samples of negatively (positively) polarised electron beams with an integrated luminosity of $105.9(73.4) \mathrm{pb}^{-1}$ and luminosity-weighted average polarisation of $P_{e}^{\text {neg }}=-0.27\left(P_{e}^{\text {pos }}=+0.30\right)$ were analysed. A detailed description of the ZEUS detector can be found elsewhere $[2,3]$. The kinematic variables $Q^{2}$ and the inelasticity, $y$, were reconstructed using the method of Jacquet-Blondel [4], which uses the information from the hadronic energy flow of the event, and were corrected for detector effects as described elsewhere [5]. The main selection criteria rely on missing transverse momentum $\left(p_{\top}\right)$, transverse energy $\left(E_{\top}\right), Q^{2}$ and $y$ by requiring $p_{\top}>11 \mathrm{GeV}$, $p_{\top} / E_{\top}>0.5, Q^{2}>200 \mathrm{GeV}^{2}$, and $y<0.9$.

\footnotetext{
${ }^{*}$ On behalf of the ZEUS Collaboration.
} 
Jets were identified in the pseudorapidity $(\eta)$ - azimuth $(\phi)$ plane of the laboratory frame using the $k_{\top}$ cluster algorithm [6] in the longitudinally invariant inclusive mode [7]. The jets have been reconstructed using the ZEUS calorimeter (CAL) and corrected for detector effects to yield jets of hadrons. Events with at least one jet of $-1<\eta^{\text {jet }}<2.5$ were retained. The final inclusive-jet sample consisted of events with at least one jet of $E_{T}^{\text {jet }}>14$ $\mathrm{GeV}$. The dijet (three-jet) sample was selected as a subset of the inclusive sample, with the requirement of an additional jet (two jets) with $E_{T}^{\text {jet }}>5 \mathrm{GeV}$. A small number of four-jet events, with one jet of $E_{\top}^{\text {jet }}>14 \mathrm{GeV}$ and at least three additional jets with $E_{\top}^{\text {jet }}>5$ were also identified in the data.

\section{Monte Carlo simulation and systematic uncertainty estimation}

Samples of MC events were generated to determine the response of the detector to jets of hadrons and to evaluate the correction factors necessary to obtain the hadron-level jet cross sections. The CC DIS events were generated using the Color Dipole Model (CDM) as implemented in the ARIADNE program [8] using the CTEQ5D [9] proton parton distribution functions. Fragmentation into hadrons was performed using the Lund string model [10] as implemented in JETSET 7.4 [11].

The following sources of systematic uncertainty were considered for the measurements of the jet cross sections: absolute energy scale of the jets and CAL, $p_{\top}$ selection cut, photoproduction background and trigger selection. The systematic uncertainties not associated with the absolute energy scale of the jets were added in quadrature to the statistical uncertainties and are shown in the figures as error bars. The uncertainty due to the absolute energy scale of the jets is shown separately as a shaded band in each figure, due to the large bin-to-bin correlation. In addition, there was an overall normalisation uncertainty of $3.5 \%$ from the luminosity determination.

\section{Results}

\subsection{Inclusive-jet cross sections}

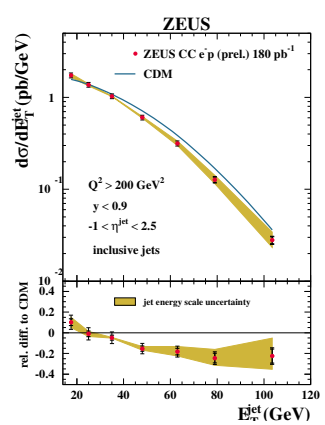

Figure 1a

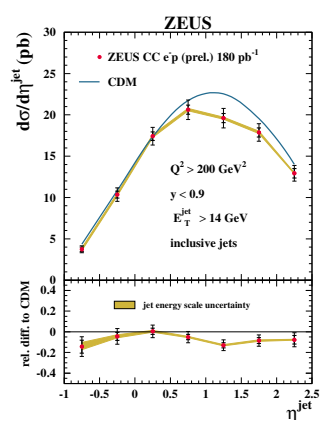

Figure 1b

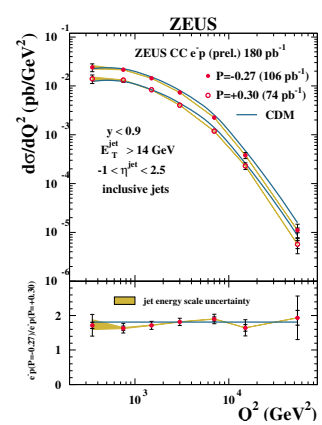

Figure 1c

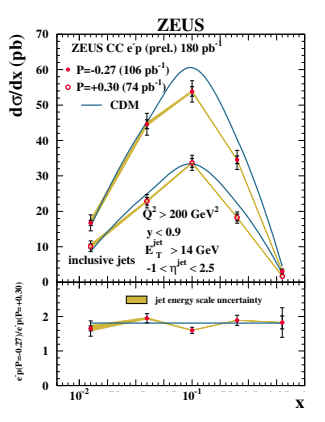

Figure 1d

Figure 1 shows the $\mathrm{CC} e^{-} p$ DIS inclusive-jet differential cross sections as functions of $E_{T}^{\text {jet }}$ (unpolarised-corrected), $\eta^{\text {jet }}$ (unpolarised-corrected), $Q^{2}$ (polarised) and $x$ (polarised). The 
unpolarised-corrected cross section for observable $A$ was obtained via $\frac{d \sigma}{d A}=\frac{N_{\mathrm{data}}^{\mathrm{unpol}}}{\mathcal{L} \cdot \Delta A} \cdot \frac{N_{\mathrm{MC}}^{\mathrm{had}}}{N_{\mathrm{MC}}^{\mathrm{det}}}$, where $N_{\text {data }}^{\text {unpol }}=\frac{N_{\text {data }}^{\text {neg }}}{1-P_{e}^{\text {Ileg }}}+\frac{N_{\text {data }}^{\text {pos }}}{1-P_{e}^{\text {pos }}}, \mathcal{L}$ is the total integrated luminosity and $\Delta A$ is the bin width.

Below the unpolarised-corrected differential cross sections with respect to $E_{\top}^{\text {jet }}$ and $\eta^{\text {jet }}$, the relative difference to $\mathrm{MC}$ is shown. Values of $E_{\top}^{\text {jet }}$ above $100 \mathrm{GeV}$ are accessible with the present statistics, and a different shape than the prediction can be seen. The measured $d \sigma / d \eta^{\text {jet }}$ has a maximum at $\eta^{\text {jet }} \sim 0.75$. For $200<Q^{2} \lesssim 1000 \mathrm{GeV}^{2}$, the distribution displays almost no dependence on $Q^{2}$. The cross sections as functions of $E_{\mathrm{T}}^{\mathrm{jet}}$ and $Q^{2}$ show a less rapid fall-off than what is observed for the same cross sections in Neutral Current DIS processes due to the presence of the massive $W$ propagator. The measured cross section in Fig.1d shows that the $x$ values accessible by the data are within the range $0.013<x<0.63$. The measured cross sections are reasonably well described for low values of $Q^{2}$ and $x$; for higher values, the data lie below the predictions. In all cases, the normalisation of the prediction is in agreement with the data.

Figures 1c and 1d show the CC negatively- and positively-polarised $e^{-} p$ DIS inclusive-jet differential cross sections as functions of $Q^{2}$ and $x$. The predictions of the leading-logarithm parton-shower MC model of CDM are compared to the data in the figure. The lower part of the figures show the ratio of the cross section for negatively- and positively-polarised electron beams, which is agreement with the predicted polarisation ratio, $\left(1-P_{e}^{\text {neg }}\right) /\left(1-P_{e}^{\text {pos }}\right)=1.81$.

The total inclusive-jet cross sections in the kinematic regime given by $Q^{2}>200 \mathrm{GeV}^{2}$, $y<0.9, E_{\top}^{\text {jet }}>14 \mathrm{GeV}$ and $-1<\eta^{\text {jet }}<2.5$ are

$$
\begin{aligned}
& \sigma_{\text {jets }}\left(P_{e}=-0.27\right)=64.42 \pm 0.96 \text { (stat.) } \pm 3.32 \text { (syst.) }{ }_{-0.55}^{+0.62} \text { (E scale) pb, } \\
& \sigma_{\text {jets }}\left(P_{e}=+0.30\right)=36.58 \pm 0.87 \text { (stat.) } \pm 1.92 \text { (syst.) }{ }_{-0.31}^{+0.35} \text { (E scale) pb, }
\end{aligned}
$$

which agree with SM predictions: $\sigma_{\text {jets }}\left(P_{e}=-0.27\right)=70 \mathrm{pb}$ and $\sigma_{\text {jets }}\left(P_{e}=+0.30\right)=39 \mathrm{pb}$.

\subsection{Dijet cross sections}

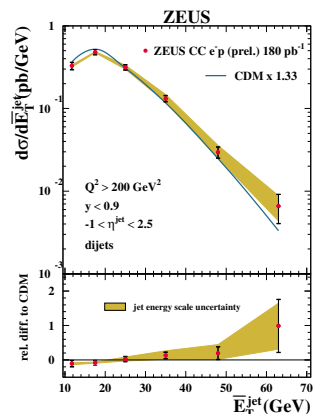

Figure 2a

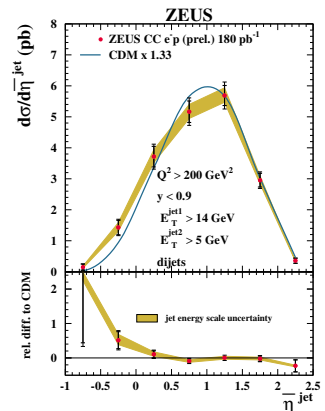

Figure $2 \mathrm{~b}$

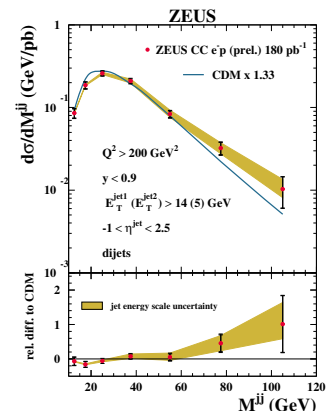

Figure 2c

Figure 2 shows the unpolarised-corrected $\mathrm{CC} e^{-} p$ DIS dijet differential cross sections as functions of mean $\eta^{\text {jet }}\left(\overline{\eta^{\text {jet }}}\right)$, mean $\left.E_{\mathrm{T}}^{\text {jet }} \overline{\left(E_{\top}^{\mathrm{jet}}\right.}\right)$, and dijet invariant mass $M^{j j}$. The measured cross section as a function of $\overline{E_{\top}^{j e t}}$ exhibits a fall-off of two orders of magnitude for 
$\overline{E_{\top}^{\text {jet }}} \gtrsim 20 \mathrm{GeV}$, but displays almost no $Q^{2}$ dependence for $200<Q^{2} \lesssim 2000 \mathrm{GeV}^{2}$. Figure 2c shows that values of $M^{j j}$ above $100 \mathrm{GeV}$ are accessible with the present statistics.

The predictions of the leading-logarithm parton-shower MC models of CDM are compared to the data in Figure 2. The predictions give a reasonable description of the shape of the data, but have been normalised to the total dijet measured cross section with a multiplicative factor of 1.33 . For $M^{j j}$, the data tend to be above the MC prediction for $M^{j j} \gtrsim 70$ $\mathrm{GeV}$.

\subsection{Three-jet cross sections}

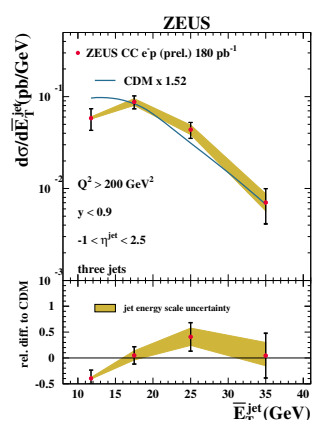

Figure $3 \mathrm{a}$

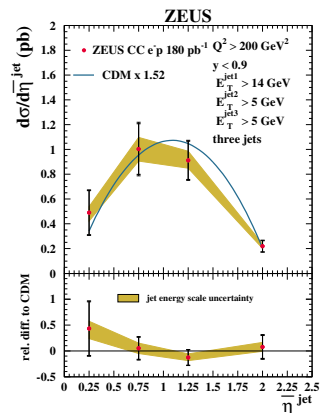

Figure $3 \mathrm{~b}$

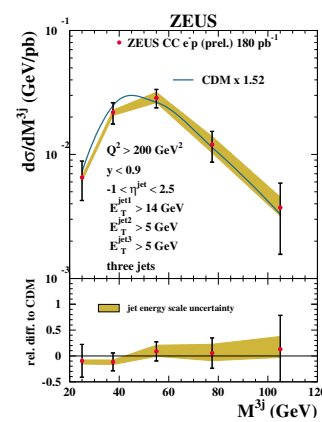

Figure 3c

Three-jet cross sections in CC DIS have been measured for the first time in ep collisions. The three-jet selected sample also contains six candidates with an extra fourth jet of $E_{\top}^{\text {jet } 4}>$ $5 \mathrm{GeV}$ in the $\eta^{\text {jet }}$ range considered.

Figure 3 shows the unpolarised-corrected CC $e^{-} p$ DIS three-jet differential cross sections

as functions of $\overline{E_{T}^{\text {jet }}}, \overline{\eta^{\text {jet }}}$, and three-jet invariant mass $\left(M^{3 j}\right)$. Values of $M^{3 j}$ above 100 $\mathrm{GeV}$ are accessible with the present statistics. The MC predictions give a good description of the shape of the data, but have been normalised to the total three-jet measured cross section with a multiplicative factor of 1.52 .

\section{References}

[1] Slides from this presentation: http://indico. cern. ch/contributionDisplay.py? contribId=224\&sessionId=6\&conf Id=9499

[2] ZEUS, M. Derrick et al., Phys. Lett. B293,465(1992)

[3] ZEUS, U.Holm(ed.), The ZEUS Detector. Status Report (unpublished), DESY (1993), available on http://www-zeus.desy.de/bluebook/bluebook.html

[4] F. Jacquet and A. Blondel, Proc. of the Study for an ep Facility for Europe, U. Amaldi (ed.), p.391. Hamburg, Germany (1979). Also in preprint DESY 79/48

[5] ZEUS, J. Breitweg et al., Eur. Phys. J. C8,367(1999)

[6] S. Catani et al., Nucl. Phys. B406,187(1993)

[7] S.D. Ellis and D.E. Soper, Phys. Rev. D48,3160(1993)

[8] L. Lönnblad, Comp. Phys. Comm. 71,15(1992)

[9] H.L. Lai et al., Eur. Phys. J. C12,375(2000)

[10] B. Andersson et al., Phys. Rep. 97,31(1983)

[11] T. Sjöstrand, Comp. Phys. Comm. 39,347(1986) 